\title{
Profils en acides gras libres, cholestérol et indices lipidiques du lait cru ovin issu de deux races algériennes (Ouled-Djellal et Rumbi) collecté en milieu steppique
}

\author{
Benalia Yabrir ${ }^{(1)}$, Moussa Labiad ${ }^{(1)}$, Abdellah Mostefaoui ${ }^{(1)}$, Ahcène Hakem ${ }^{(1)}$, \\ Yacine Titouche ${ }^{(1)}$, Frédéric Gaucheron ${ }^{(2)}$, Abderrahmane Mati ${ }^{(3)}$ \\ (1) Université Ziane Achour. Faculté des Sciences de la Nature et de la Vie. Laboratoire d'Exploration et Valorisation des \\ Écosystèmes steppiques. Route de Moudjbara. BP 3117.17000 Djelfa (Algérie).E-mail : byabrir@yahoo.fr \\ (2) Agrocampus Ouest Rennes. INRA. UMR 1253. Laboratoire Science et Technologie du Lait et de l'Oeuf (STLO). 65, rue \\ de Saint-Brieuc. FR-35042 Rennes cedex (France). \\ (3) Université Mouloud Mammeri de Tizi-Ouzou. Faculté des Sciences biologiques et des Sciences agronomiques. \\ Département de Biologie. Laboratoire de Biochimie analytique et Biotechnologies. BP 17 RP. 15000 Tizi-Ouzou (Algérie).
}

Reçu le 11 janvier 2015, accepté le 26 aout 2015.

Description du sujet. Cet article vise à caractériser la matière grasse de lait cru ovin collecté localement en milieu steppique algérien, très apprécié pour la production du smen (beurre traditionnel).

Objectifs. L'objectif de cette étude consiste à étudier le profil en acides gras libres et en cholestérol ainsi que les indices lipidiques du lait cru ovin issu de deux races locales algériennes (Ouled-Djellal et Rumbi).

Méthode. Le lait est collecté à un stade moyen de lactation auprès de 20 brebis de chaque race. La matière grasse est extraite selon la méthode Rose-Gotllieb. Les proportions en acides gras (AG) libres majeurs sont déterminées par chromatographie en phase gazeuse.

Résultats. Les acides palmitique $(24,4 \% \pm 3,3)$, oléique $(24,2 \% \pm 4,6)$, stéarique $(10,8 \% \pm 3,5)$, myristique $(10,4 \% \pm 1,9)$ et caprique $(8,3 \% \pm 3,5)$ représentent la majorité des acides gras présents en totalisant $78,1 \%$ des AG totaux. Les proportions en AG saturés sont plus importantes que celles des AG insaturés $(69,6 \% \pm 5,3 v s 30,8 \% \pm 5,1)$. Les AG monoinsaturés l'emportent sur les polyinsaturés $(26,3 \% \pm 4,7 v s 4,5 \% \pm 1,1)$. Le lait de la race Ouled-Djellal est plus riche $(p<0,05)$ en AG à chaine courte que celui de la race Rumbi. Ce dernier est plus riche en AG à chaine moyenne, bien que la différence ne soit significative que pour les acides palmitique et palmitoléique $(p<0,05)$. Parmi les AG à chaine longue, seuls les acides stéarique $(10,88 \% \pm 2,83$ vs $12,34 \% \pm 2,75 ; p<0,01)$ et arachidique $(0,26 \% \pm 0,18 v s 0,35 \% \pm 0,09 ; p<0,05)$ sont influencés par la race.

Conclusions. Les résultats obtenus montrent une richesse plus élevée en AG du lait de la race Rumbi, comparativement à celui de la race Ouled-Djellal. Ce lait peut être valorisé en technologie pour fabriquer le beurre traditionnel et aussi générer une économie locale intéressante.

Mots-clés. Lait de brebis, race indigène, acides gras, teneur en lipides, cholestérol, Algérie.

Free fatty acid profiles and cholesterol and lipid indexes of raw ewe's milk from two Algerian breeds (Ouled-Djellal and Rumbi), collected in the steppe area

Description of the subject. This article aims to characterize the fat content of raw ewe's milk, collected locally in the Algerian central steppe, an area highly appreciated for its production of smen (traditional butter).

Objectives. The objective of this study was to investigate the free fatty acid profile and the cholesterol and lipid indexes of raw ewe's milk from two Algerian local breeds (Ouled-Djellal and Rumbi).

Method. Milk was collected from 20 ewes of each breed three times during the middle stage of lactation. The milk fat was extracted using the Rose-Gottlieb method. Fatty acids were analyzed by gas chromatography.

Results. Palmitic $(24.4 \% \pm 3.3)$, oleic $(24.2 \% \pm 4.6)$, stearic $(10.8 \% \pm 3.5)$, myristic $(10.4 \% \pm 1.9)$ and capric $(8.3 \% \pm 3.5)$ acids dominated and accounted for $78.1 \%$ of the total fatty acids. Saturated fatty acids predominated compared to unsaturated fatty acids $(69.6 \% \pm 5.3 v s 30.8 \% \pm 5.1)$, and monounsaturated fatty acids outweighed the polyunsaturated $(26.3 \% \pm 4.7 v s$ $4.5 \% \pm 1.1)$. The Ouled-Djellal milk had a higher short-chain fatty acid content than the Rumbi milk $(p<0.05)$. On the other 
hand, the Rumbi milk was richer in medium chain fatty acids. The two types of milk differed significantly regarding their palmitic and palmitoleic acid content $(p<0.05)$. Within the long chain of fatty acids, only the stearic acid $(10.88 \% \pm 2.83 v s$ $12.34 \% \pm 2.75 ; p<0.01)$ and the arachidic acid $(0.26 \% \pm 0.18$ vs $0.35 \% \pm 0.09 ; p<0.05)$ were influenced by the breed.

Conclusions. Results showed that milk from the Rumbi ewe was richer in fatty acids than the milk from the Ouled-Djellal and that Rumbi ewe's milk may be of value in dairy technology due to the socio-economic impact that it can generate.

Keywords. Ewe milk, land races, fatty acids, lipid content, cholesterol, Algeria.

\section{INTRODUCTION}

Le lait de brebis est réputé pour sa richesse en matière grasse dont la teneur moyenne estestimée à $70 \mathrm{~g} \cdot \mathrm{l}^{-1}$. Cette dernière constitue un des critères de base de paiement du lait à la qualité (Pirisi et al.,2007) et contribue également aux qualités nutritionnelles et organoleptiques des produits qui en découlent (Scintu et al., 2007). La matière grasse varie en fonction de l'alimentation (qualité et quantité de l'aliment) et d'autres facteurs comme la génétique, le stade de lactation, la parité, la saison, etc. (Perea et al., 2000 ; Lock et al., 2005 ; Sanz Sampelayo et al., 2007 ; Legarto et al., 2014). Elle est constituée essentiellement de triglycérides (98\%). Les diglycérides, les monoglycérides et les acides gras (AG) libres sont naturellement présents en faibles quantités, mais leur proportion peut augmenter en cas de lipolyse (Chilliard et al., 1984). De nombreux autres composés (phospholipides, cholestérol, vitamines) sont également présents, mais à des teneurs plus réduites.

Comme la matière grasse, les AG influencent les propriétés technologiques et organoleptiques des produits laitiers (proportions variables d'AG, sensibilité à l'oxydation, etc.) (Schmidely et al., 2001). Cette composition peut varier en fonction de plusieurs facteurs tels que l'alimentation (Cieslak et al., 2010 ; Abbeddou et al., 2011 ; Legarto et al., 2014), l'environnement (Collomb et al., 2006) et les facteurs génétiques et/ou physiologiques comme la race, la parité et le stade de lactation (Carta et al., 2008 ; De La Fuente et al., 2009; Mierlita et al., 2011a ; Mierlita et al., 2011b ; Boichard et al., 2014 ; Gelé et al., 2014). L'étude du profil en AG du lait de brebis a fait l'objet d'un certain nombre de travaux de recherches (Lock et al., 2005 ; Biondi et al., 2008 ; Carta et al., 2008 ; Castro et al., 2009 ; Mierlita et al., 2011a; Mierlita et al., 2011b), avec souvent des données différentes selon les auteurs.

En milieu steppique algérien, le lait de brebis est consommé frais ou transformé à l'échelle artisanale en smen (beurre traditionnel). Ce dernier, très apprécié par les populations, est vendu 25 euros le litre et constitue une source de revenu complémentaire pour les producteurs. À notre connaissance, le lait entier n'a fait l'objet que de très peu d'investigation (Yabrir et al., 2013 ; Yabrir et al., 2014). Ainsi, la présente étude vise à mieux caractériser ce lait à travers les deux principales races, Ouled-Djellal et Rumbi, dominantes par leurs effectifs et concentrées en milieu steppique algérien. Cette caractérisation consiste à extraire la matière grasse pour en étudier sa composition et notamment le profil en acides gras libres, la concentration en cholestérol et les indices de saponification et d'iode. L'effet de la race est également discuté.

\section{MATÉRIEL ET MÉTHODES}

\section{1. Échantillonnage}

L'étude a été conduite sur 120 échantillons de lait cru individuel. Les échantillons provenaient de brebis de deux races: Ouled-Djellal (OD) (60 échantillons), Rumbi (RB) (60 échantillons), conduites dans un même troupeau. L'élevage fut conduit en mode semi-intensif où l'alimentation était basée essentiellement sur les parcours steppiques. Les brebis (20 de chaque race) étaient traites le soir après le retour du troupeau à la bergerie. Les prélèvements étaient effectués trois fois de suite à raison de 15 à 20 jours d'intervalle pendant le stade moyen de lactation. Les laits collectés furent transportés au laboratoire dans des glacières à $4{ }^{\circ} \mathrm{C}$. La durée entre la traite et l'extraction de la matière grasse n'excéda pas $24 \mathrm{~h}$.

\subsection{Extraction de la fraction lipidique}

La détermination de la matière grasse correspond à un dosage par pesée après extraction éthéroammoniacale selon la méthode Rose-Gottlieb (AOAC, 1998). L'extraction de la matière grasse fut réalisée par additions successives d'ammoniaque, d'alcool éthylique à $95 \%$, d'éther éthylique et d'éther de pétrole. L'extraction fut répétée deux fois de suite.

\subsection{Déterminations des indices}

La détermination des indices permet de caractériser la matière grasse extraite en évaluant la longueur des chaines d'AG, la présence des AG libres et le degré d'insaturation. Les indices de saponification (I $)_{s}$ et d'iode $\left(\mathrm{I}_{\mathrm{i}}\right)$ furent déterminés par titrage (Gavrilovie et al., 1996). L'indice d'athérogénécité fut calculé selon Ulbricht et al. (1991). 


\subsection{Détermination des profils en AG libres}

Préparation des esters méthyliques d'AG. La formation des esters méthyliques d'AG est une étape préalable à la chromatographie, elle a été opérée conformément au protocole décrit par Mordret (1992). Elle consiste en une méthanolyse en milieu alcalin, au cours de laquelle il y a saponification des AG libres, méthanolyse des triglycérides et qui est suivie d'une méthanolyse en milieu acide transformant les savons en AG puis en esters. Les esters méthyliques ainsi formés furent extraits par un solvant apolaire puis analysés par chromatographie en phase gazeuse.

Conditions chromatographiques. Le chromatographe utilisé (Trace GC, Thermo Finnigam, Rodano, Italie) était équipé d'une colonne capillaire FFAP (Free Fatty Acid Phase) de $30 \mathrm{~m}$ de long et de $0,25 \mathrm{~mm}$ de diamètre interne. L'azote, utilisé comme gaz vecteur, avait un débit de $5,4 \mathrm{ml} \cdot \mathrm{min}^{-1}$ et une pression initiale de $150 \mathrm{Kpa}$. La détection était assurée par un détecteur à ionisation de flamme. Pour l'intégration des surfaces des pics chromatographiques, le logiciel Chrom-Card ${ }^{\mathrm{TM}}$ a été utilisé. Les conditions chromatographiques étaient les suivantes :

$-0,4 \mu 1$ de chaque extrait est injecté en mode «on column »,

- température de l'injecteur : $210^{\circ} \mathrm{C}$,

- température du détecteur : $230^{\circ} \mathrm{C}$,

- température de la colonne : $45^{\circ} \mathrm{C}$ pendant $3 \mathrm{~min}, \quad 20^{\circ} \mathrm{C} \cdot \mathrm{min}^{-1}$ jusqu'à $190^{\circ} \mathrm{C}$, cette température est maintenue pendant $30 \mathrm{~min}$.

\subsection{Dosage du cholestérol}

Le cholestérol a été dosé par spectrophotométrie visible à une longueur d'onde de $550 \mathrm{~nm}$, comme décrit par Naudet et al. (1986) et Barreto (2005). Le principe repose sur une réaction colorée spécifique des $3 \beta$-hydroxystéroides possédant une double liaison en 5-6.En présence de cholestérol, le réactif spectral de Liebermann développe une coloration verte stable au bout de 25 min à l'obscurité. En parallèle, une courbe d'étalonnage du cholestérol a été établie.

\section{RÉSULTATS}

\subsection{Profil en AG libres}

Les proportions moyennes en AG des échantillons du lait cru ovin des deux races sont indiquées dans le tableau 1. À la lecture des chromatogrammes, dont un exemple type est reporté sur la figure 1, on note l'existence de 18 pics dont 5 avec une représentation majeure pour l'acide palmitique $(24,4 \% \pm 3,3)$, l'acide oléique $(24,2 \% \pm 4,6)$, l'acide stéarique $(10,8 \% \pm 3,5)$, l'acide myristique $(10,4 \%$

Tableau 1. Concentrations des acides gras libres (en \%) du lait cru ovin - Free fatty acids content (in \%) of ewe's raw milk.

\begin{tabular}{|c|c|c|c|c|}
\hline \multirow[t]{2}{*}{ Acide gras } & \multicolumn{2}{|c|}{ Race (moyenne \pm écart-type) } & \multirow[t]{2}{*}{$p$} & \multirow{2}{*}{$\begin{array}{l}\text { Moyenne } \\
\pm \text { écart-type }\end{array}$} \\
\hline & Ouled-Djellal & Rumbi & & \\
\hline $\mathrm{C} 4$ & $3,05 \pm 1,76$ & $2,51 \pm 0,63$ & Ns & $2,91 \pm 1,21$ \\
\hline C6 & $2,82 \pm 1,11$ & $2,21 \pm 0,38$ & $*$ & $2,75 \pm 1,20$ \\
\hline $\mathrm{C} 8$ & $2,93 \pm 1,05$ & $2,26 \pm 0,50$ & $*$ & $2,90 \pm 1,33$ \\
\hline $\mathrm{C} 10$ & $8,24 \pm 2,37$ & $6,73 \pm 2,02$ & $*$ & $8,30 \pm 3,47$ \\
\hline C10: 1 & $0,23 \pm 0,17$ & $0,19 \pm 0,08$ & $* *$ & $0,24 \pm 0,12$ \\
\hline $\mathrm{C} 12$ & $4,43 \pm 2,58$ & $3,86 \pm 1,15$ & $*$ & $4,49 \pm 1,53$ \\
\hline $\mathrm{C} 14$ & $10,14 \pm 2,06$ & $10,72 \pm 2,07$ & Ns & $10,42 \pm 1,96$ \\
\hline $\mathrm{C} 15$ & $1,06 \pm 0,27$ & $1,13 \pm 0,24$ & Ns & $1,07 \pm 0,28$ \\
\hline $\mathrm{C} 16$ & $24,25 \pm 3,06$ & $25,85 \pm 2,84$ & $*$ & $24,41 \pm 3,30$ \\
\hline C16: 1 & $1,43 \pm 0,55$ & $1,36 \pm 0,22$ & $*$ & $1,42 \pm 0,30$ \\
\hline $\mathrm{C} 17$ & $0,83 \pm 0,24$ & $0,87 \pm 0,18$ & Ns & $0,83 \pm 0,22$ \\
\hline C17: 1 & $0,35 \pm 0,11$ & $0,35 \pm 0,09$ & Ns & $0,35 \pm 0,10$ \\
\hline $\mathrm{C} 18$ & $10,88 \pm 2,83$ & $12,34 \pm 2,75$ & $* *$ & $10,84 \pm 3,53$ \\
\hline C18: 1 & $24,34 \pm 4,11$ & $24,94 \pm 4,06$ & Ns & $24,18 \pm 4,59$ \\
\hline C18: 2 & $3,54 \pm 0,88$ & $3,35 \pm 0,57$ & Ns & $3,47 \pm 0,74$ \\
\hline C18: 3 & $1,03 \pm 0,69$ & $0,83 \pm 0,26$ & Ns & $1,00 \pm 0,47$ \\
\hline $\mathrm{C} 20$ & $0,26 \pm 0,18$ & $0,35 \pm 0,09$ & $*$ & $0,27 \pm 0,13$ \\
\hline C20: 1 & $0,15 \pm 0,04$ & $0,17 \pm 0,07$ & Ns & $0,15 \pm 0,06$ \\
\hline $\mathrm{AGS}^{\mathrm{a}}$ & $69,37 \pm 4,95$ & $69,21 \pm 4,57$ & Ns & $69,64 \pm 5,28$ \\
\hline $\mathrm{AGI}^{\mathrm{b}}$ & $31,08 \pm 4,90$ & $31,17 \pm 4,48$ & Ns & $30,81 \pm 5,15$ \\
\hline $\mathrm{AGMI}^{\mathrm{c}}$ & $26,51 \pm 3,93$ & $26,99 \pm 4,13$ & Ns & $26,33 \pm 4,70$ \\
\hline $\mathrm{AGP}^{\mathrm{d}}$ & $4,57 \pm 0,97$ & $4,17 \pm 0,66$ & Ns & $4,48 \pm 1,05$ \\
\hline$\sum \mathrm{AGCC}^{\mathrm{e}}$ & $21,71 \pm 4,13$ & $17,76 \pm 3,60$ & $*$ & $21,57 \pm 7,88$ \\
\hline$\sum$ AGCMf & $38,06 \pm 4,04$ & $40,27 \pm 4,19$ & $*$ & $38,49 \pm 4,58$ \\
\hline$\sum \mathrm{AGCL}^{\mathrm{g}}$ & $40,21 \pm 6,06$ & $41,96 \pm 6,84$ & Ns & $39,92 \pm 7,61$ \\
\hline $\mathrm{IA}^{\mathrm{h}}$ & $2,40 \pm 0,69$ & $2,36 \pm 0,73$ & Ns & $2,38 \pm 0,68$ \\
\hline
\end{tabular}

Ns : non significatif - not significant $; *$ : $p<0,05-\mathrm{p}<0.05$;

$* *: p<0,01-\mathrm{p}<0.01 ;{ }^{a}$ : acides gras saturés - saturated fatty acids $;{ }^{\mathrm{b}}$ : acides gras insaturés - unsaturated fatty acids $;^{\mathrm{c}}$ : acides gras monoinsaturés - monounsaturated fatty acids $;{ }^{\mathrm{d}}$ : acides gras polyinsaturés - polyunsaturated fatty acids ${ }^{\mathrm{e}}:$ : somme des acides gras à chaines courtes - sum of short chain fatty acids $(\mathrm{C} 4: 0-\mathrm{C} 12: 0)$; ${ }^{\mathrm{f}}$ : somme des acides gras à chaines moyennes - sum of medium chain fatty acids (C14: $0-\mathrm{C} 17: 1) ;^{\mathrm{g}}$ : somme des acides gras à chaines longues - sum of long chain fatty acids $(\geq \mathrm{C} 18: 0) ;{ }^{\mathrm{h}}:$ indice d'athérogénécité - atherogenic index (C12: $0+4 * \mathrm{C} 14: 0+\mathrm{C} 16: 0) / \Sigma$. 


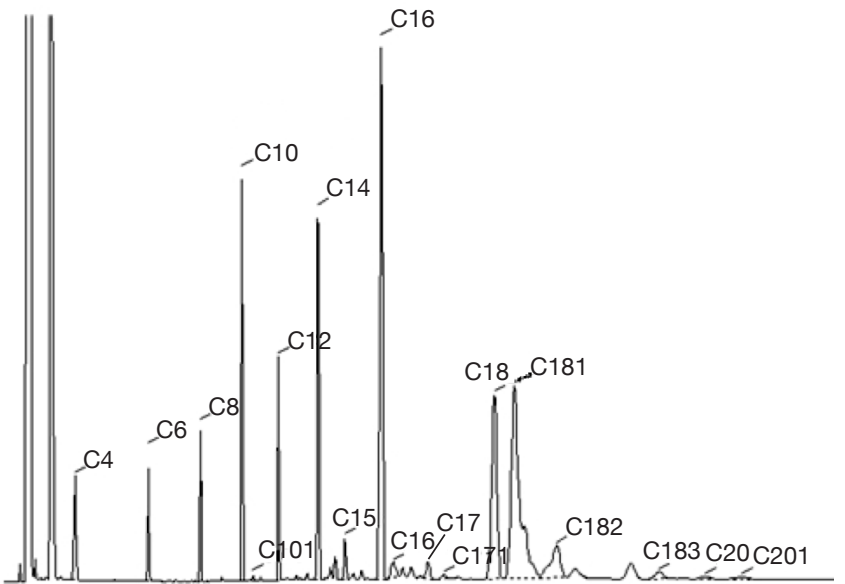

Figure 1. Exemple type d'un chromatogramme (CPG) pour un échantillon de lait cru ovin - Chromatographic profile of ewe's raw milk sample.

$\pm 1,9)$ et l'acide caprique $(8,3 \% \pm 3,5)$. Ces AG correspondaient à 78,15\% des AG totaux.

Les AG saturés étaient plus importants que les AG insaturés $(69,6 \%$ vs 30,8\%). Parmi les AG saturés, ceux dont la chaine hydrocarbonée est supérieure ou égale à $14 \mathrm{C}$ étaient majoritaires $(68 \%$ des AG saturés totaux) avec une prédominance de l'acide palmitique $(35,1 \%)$ suivi des acides stéarique et myristique qui avaient des proportions respectives de $15,6 \%$ et $14,9 \%$ par rapport aux AG saturés totaux. Les AG saturés à chaine courte (C4-C12) totalisaient un pourcentage de $21,6 \%$ et représentaient $30,7 \%$ des AG saturé totaux. Dans ce groupe, l'acide caprique était le constituant majoritaire avec un taux de $8,3 \% \pm 3,5$ par rapport au total des AG, suivi des acides laurique, butyrique et caprylique.

Pour ce qui est des AG insaturés, les monoinsaturés étaient plus importants que les AG polyinsaturés (26,3\% vs 4,5\%). L'acide oléique prédominait avec un pourcentage moyen de $24,2 \%$, alors que les autres AG monoinsaturés représentaient $2,1 \%$ des AG totaux.

L'indice d'athérogénécité lié aux AG saturés en $\mathrm{C} 12, \mathrm{C} 14$ et $\mathrm{C} 16$ variait entre 2,36 (race RB) et 2,40 (race OD) pour une moyenne de 2,38.

\subsection{Effet de la race}

Les concentrations de divers AG des laits crus ovins analysés différaient d'une race à l'autre, parfois significativement (Tableau 1). Tous les AG à chaine courte, à l'exception de l'acide butyrique, avaient des concentrations plus importantes dans les laits de la race OD.

$\mathrm{Au}$ contraire, le lait de la race $\mathrm{RB}$ était plus riche en $A G$ à chaine moyenne, bien que ces derniers ne différaient significativement que par rapport aux acides palmitique et palmitoléique $(p<0,05)$. Parmi les AG à chaine longue, seuls les acides stéarique $(p<0,01)$ et arachidique $(p<0,05)$ étaient influencés par la race, avec une richesse importante pour la race RB.

Les proportions en $A G$ saturés et $A G$ insaturés n'étaient pas différentes selon la race.

\subsection{Indices lipidiques et teneur en cholestérol}

Les valeurs d'indices de saponification et d'iode sont reportées dans le tableau 2 pour le lait cru ovin issu des deux races. Les valeurs étaient plus élevées pour le lait issu de la race OD. Cependant, l'effet race n'était pas significatif.

La teneur en cholestérol variait de 113 à $592 \mathrm{mg} \cdot 100 \mathrm{~g}^{-1}$ de $\mathrm{MG}$ avec une moyenne de $362 \pm 163$ (Tableau 3). Les valeurs extrêmes furent observées dans la matière grasse du lait de la race RB. Cependant, la race n'a pas eu d'effet significatif sur la teneur en cholestérol.

\section{DISCUSSION}

Ces résultats constituent une première caractérisation lipidique du lait cru ovin collecté en milieu steppique algérien. Le profil lipidique constitue une source d'informations utiles à connaitre, notamment pour appréhender les caractéristiques organoleptiques, nutritionnelles et technologiques du smen.

\subsection{Profil en acides gras}

À l'exception des résultats obtenus par Lock et al. (2005) et Biondi et al. (2008) où il est reporté que

Tableau 2. Indices de la matière grasse du lait cru ovin - Indexes of ewe's raw milk fat.

\begin{tabular}{lrrrrr}
\hline Indice & \multicolumn{2}{c}{ Race } & & $\boldsymbol{p}$ & Moyenne \\
\cline { 2 - 3 } & OD & RB & & \\
\hline $\mathrm{I}_{\mathrm{s}}$ & 231 & 220 & Ns & 225 \\
$\mathrm{I}_{\mathrm{i}}$ & 44 & 32 & Ns & 38 \\
\hline
\end{tabular}

Ns : non significatif - not significant; $\mathrm{I}_{\mathrm{s}}$ : indice de saponification - saponification value; $\mathrm{I}_{\mathrm{i}}$ : indice d'iode - iodine value.

Tableau 3. Teneur en cholestérol $\left(\mathrm{mg} \cdot 100 \mathrm{~g}^{-1}\right.$ de matière grasse) - Cholesterol content ( $\mathrm{mg} \cdot 100 \mathrm{~g}^{-1}$ of fat).

\begin{tabular}{lllll}
\hline & Moyenne & Écart-type & Min & Max \\
\hline Les deux races & 362 & 163 & 113 & 592 \\
Ouled-Djellal & 319 & 81 & 184 & 437 \\
Rumbi & 405 & 219 & 113 & 592 \\
\hline
\end{tabular}


l'acide butyrique domine par rapport à l'acide caprique, l'importance quantitative des AG en C16, C18: 1, C18, C14 et C10 est aussi observée par plusieurs auteurs (Zhang et al., 2006 ; Carta et al., 2008 ; Talpur et al., 2008 ; Castro et al., 2009 ; Hilali et al., 2011 ; Mierlita et al., 2011a ; Mierlita et al., 2011b).

L'importance des AG saturés par rapport aux AG insaturés a été observée aussi bien pour le lait de brebis (59,3 à 74,3\% vs 22,8 à 39,2\%) (Biondi et al., 2008 ; Carta et al., 2008 ; Mierlita et al., 2011a) que pour le lait de vache (67 à $69 \%$ vs 30,6\% à 34,3\%) (Moate et al., 2007 ; Gelé et al., 2014).

Dans le groupe des AG à chaine courte, les teneurs moyennes étaient dans l'ordre croissant suivant: $\mathrm{C} 10<\mathrm{C} 12<\mathrm{C} 4<\mathrm{C} 8$. Cette tendance était conforme à d'autres études faites également sur le lait de brebis (Zhang et al., 2006 ; Carta et al., 2008 ; Hilali et al., 2011 ; Mierlita et al.,2011a). Lock et al.(2005) et Biondi et al. (2008) ont montré que l'acide butyrique était plus important quantitativement que l'acide caprique (4,2 et $11,3 \%$ vs 3,5 et 8,3\% respectivement). La proportion en acide butyrique estimée à $2,91 \%$ était inférieure à celle du lait de vache (Moate et al., 2007 ; Talpur et al., 2008 ; Gelé et al., 2014), ce qui est en contradiction avec les résultats de Barlowska et al. (2011), indiquant que la teneur élevée en acide butyrique est l'une des caractéristiques du lait de brebis par rapport à celui de la vache ou de la chèvre. Bien que les AG saturés n'aient pas une importance nutritionnelle (Gnadig et al., 2001), les teneurs des acides à chaine courte et moyenne en font une matière grasse très digestible (Paccalin et al., 1986). Par ailleurs, ils participent à l'arôme des produits laitiers du fait de leur libération sous l'action des lipases naturelles ou microbiennes (Chilliard et al., 1984).

L'importance quantitative des AG monoinsaturés par rapport aux AG polyinsaturés était comme attendu et conforme aux résultats obtenus aussi bien pour le lait de brebis (Mierlita et al., 2011b) que pour celui de la vache (Gelé et al., 2014). La teneur moyenne de l'acide oléique déterminée était soit supérieure à celle des laits d'autres brebis dans divers pays (Goudjil et al., 2004 ; Zhang et al., 2006 ; Valvo et al., 2007 ; Biondi et al., 2008 ; Carta et al., 2008 ; Castro et al., 2009 ; De La Fuente et al., 2009; Hilali et al., 2011 ; Mierlita et al., 2011a ; Gelé et al., 2014), soit inférieure (Signorelli et al.,2008; Talpur et al., 2009), soit similaire (Perea et al., 2000). Elle était aussi supérieure à celle du lait de vache (20-22 \%, Moate et al., 2007 ; Gelé et al., 2014). Les proportions des autres AG monoinsaturés, en totalisant $2,15 \%$ des AG totaux, étaient supérieures à celles évoquées pour le lait de brebis (Lock et al., 2005 ; Carta et al., 2008 ; Castro et al., 2009 ; Hilali et al., 2011 ; Mierlita et al., 2011a) mais inférieures à d'autres de la même espèce (Zhang et al., 2006 ; Biondi et al., 2008) et de l'espèce bovine (Moate et al., 2007 ; Gelé et al., 2014).
L'indice d'athérogénécité a été défini pour la première fois par Ulbricht et al. (1991) pour mettre en évidence l'effet négatif des AG saturés en C12, C14 et C16. La valeur obtenue de cet indice $(2,38)$ était inférieure à celle obtenue par Castro et al. (2009), estimée à 2,94, mais supérieure à celles trouvées par Gomez-Cortes et al. (2009) évaluée à 1,53 et Cieslak et al. (2010) estimée entre 1,3 et 1,4. Cet indice est estimé à 2,55 pour le lait de vache et 2,88 pour le lait de chèvre (Cieslak et al., 2010).

\subsection{Effet de la race}

Les facteurs qui affectent la composition en $\mathrm{AG}$ du lait de brebis ont fait l'objet d'un travail de recherche publié par De La Fuente et al. (2009). Parmi ces facteurs, l'alimentation est le facteur le plus étudié (Morand-Fehr et al., 2007 ; Sanz Sampelayo et al., 2007 ; Biondi et al., 2008 ; Castro et al., 2009 ; Gomez-Cortes et al., 2009). À un degré moindre, la saison (Perea et al., 2000 ; Talpur et al., 2008), bien que celle-ci soit liée au régime alimentaire des brebis au pâturage (De La Fuente et al., 2009), améliore la qualité nutritionnelle du lait (Valvo et al., 2007). Les facteurs liés à l'animal sont moins étudiés. On cite, à titre d'exemples, le stade de lactation, l'âge des brebis, le sexe de l'agneau et le nombre de portées (Carta et al., 2008 ; De La Fuente et al., 2009). D'autres travaux ont mis l'accent sur l'effet de la race sur le profil lipidique de la matière grasse laitière ovine (Mihaylova et al., 2004 ; Barbosa et al., 2007 ; Signorelli et al., 2008 ; Talpur et al., 2009 ; Mierlita et al., 2011a ; Mierlita et al., 2011b ; Gerchev et al., 2012).

L'effet de la race sur les AG à chaine courte, à l'exception de l'acide butyrique, a été aussi observé par Talpur et al. (2009) et Mierlita et al. (2011a) en comparant les races Kachi et Kooka pakistanaises et Spanca et Turcana roumaines, respectivement. Les races Spanca et Kachi sont les plus riches en AG à chaine courte. La race Spanca se rapproche de la race $\mathrm{OD}$, surtout pour les taux des acides caprö̈que et caprylique, alors que la race Kachi est similaire pour l'acide caproïque. D'autre part, la race OD se rapproche de la race Turcana pour ce qui est des acides caprique et laurique et présente des taux supérieurs à ceux de la race Kooka. Selon Mihaylova et al. (2004), le lait issu de la race Karakachan était plus riche en AG à chaine courte que la race Tsigay. Contrairement à ce constat, Barbosa et al. (2007) n'avaient trouvé aucun effet de la race sur ces concentrations en comparant les races Assaf et Churra d'une part, et les races Awassi, Chura et Lacaune d'autre part.

Pour ce qui est des AG à chaine moyenne, l'effet de la race est disparate et parfois contradictoire. Ainsi, contrairement à nos résultats, Talpur et al. (2009) ont observé un effet uniquement pour l'acide myristique ; 
Mierlita et al. (2011a) ont trouvé un effet sur les acides myristique et palmitique, alors que Barbosa et al. (2007) ont reporté l'effet de la race sur les acides palmitique et margarique.

Notre résultat est en contradiction avec celui de Mierlita et al. (2011a) qui reportent qu'aucun AG à longue chaine n'est influencé par la race, à l'exception des acides oléique (C18:1Cis9) et vaccénique (C18:1 Trans-11). Aussi, Mihaylova et al. (2004) ont constaté l'effet de la race sur ces deux acides : le lait issu de la race Tsigay était plus riche en acide oléique comparé à celui de la race Karakachan et ce dernier était plus riche en acide vaccénique. D'autre part, Talpur et al. (2009) relatent l'effet de la race sur les AG stéarique, oléique, vaccénique. La teneur en acide stéarique de la race OD $(10,9 \%)$ est supérieure à celle de la race Kooka, celle de la race $\mathrm{RB}(12,3 \%)$ à celle de la race Kachi (Talpur et al., 2009). Elles étaient aussi supérieures aux races Altamurana, Gentile di Puglia et Sarda (Signorelli et al., 2008), Spanca et Turcana (Mierlita et al., 2011a), mais inférieures aux races Srednostaroplaninska et Tetevenska (Gerchev et al., 2012). Pour ce qui est de la teneur en acide arachidique, le lait de la race $\mathrm{RB}$ se rapproche des laits issus des races Spanca et Turcana (Mierlita et al., 2011a) et Assaf et Churra (Barbosa et al., 2007), alors que la race OD se rapproche des races Awassi, Churra et Lacaune (Barbosa et al., 2007).

L'absence d'effet de la race sur les catégories d'AG contredit celui reporté par Talpur et al. (2009) et Mierlita et al. (2011a). À l'inverse, nos résultats étaient en accord avec ceux de Signorelli et al. (2008) pour tous les groupes et ceux de Mihaylova et al. (2004) et Barbosa et al. (2007) pour tous les groupes, exceptés les AG polyinsaturés.

L'effet de la race sur la composition de la matière grasse est aussi observé chez la vache (Gelé et al., 2014). Les facteurs qui affectent la composition en $\mathrm{AG} \mathrm{du}$ lait des ruminants (vache, chèvre et brebis) ont également fait l'objet d'une revue de synthèse par Legarto et al. (2014). Les plus importants de ces facteurs sont liés à l'alimentation et à l'animal.

\subsection{Indices lipidiques et teneur en cholestérol}

L'indice de saponification (I) est lié à la grandeur moléculaire moyenne des AG, alors que l'indice d'iode $\left(\mathrm{I}_{\mathrm{i}}\right)$ est en relation avec le degré d'insaturation des AG. Les moyennes déterminées pour les $\mathrm{I}_{\mathrm{i}}$ et $\mathrm{I}_{\mathrm{s}}$ (Tableau 2) étaient pour le premier légèrement supérieures (38 vs 20-35) et pour le second légèrement inférieures (225 $v s$ 230-245) à celles évoquées par Assenat (1985) et Park et al. (2007) pour le lait de brebis. Par contre, elles étaient similaires aux valeurs observées pour le lait de vache (32-42 pour l' $I_{i}$ et 220-232 pour l' $I_{s}$ ) (Assenat, 1985).
Nous n'avons pas trouvé d'effet significatif de la race sur la teneur en cholestérol (Tableau 3), alors que Goudjil et al. (2003) ont rapporté un effet race. Selon Chambon (1992), la teneur en stérol de la matière grasse laitière est estimée à $300 \mathrm{mg} \cdot 100 \mathrm{~g}^{-1}$ de corps gras dont $99 \%$ sont constitués de cholestérol. Pour Boutonnier et al. (1990), la teneur en stérol varie de 200 à $386 \mathrm{mg} \cdot 100 \mathrm{~g}^{-1}$ avec une teneur en cholestérol libre estimée à $300 \mathrm{mg} \cdot 100 \mathrm{~g}^{-1}$. Paccalin et al. (1986) rapportent une teneur en cholestérol similaire, en précisant que le lait en contient $105 \mathrm{mg} \cdot \mathrm{l}^{-1}$. Pour le lait de brebis, la teneur en cholestérol varie de 15 à $30 \mathrm{mg}$ pour $100 \mathrm{ml}$ de lait (Assenat, 1985). Goudjil et al. (2003) rapportent une teneur de $288 \mathrm{mg} \cdot 100 \mathrm{~g} \mathrm{~g}^{-1}$ de matière grasse.

\section{CONCLUSIONS}

Cette étude de composition en matière grasse de lait issu de deux races ovines (Rumbi et Ouled-Djellal) a montré des différences qualitative et quantitative notamment au niveau des AG. Le lait de la race Rumbi était plus riche en AG comparativement au lait de la race Ouled-Djellal avec un effet race significatif pour certains paramètres. Par contre, les concentrations en cholesterol, ainsi que les indices $I_{s}$ et $I_{i}$, n'étaient pas significativement différents entre les deux races considérées. Ces résultats étaient globalement en accord avec la littérature, avec parfois quelques différences selon les auteurs. En perspective, nous envisageons d'étudier le comportement de ces laits en technologie beurrière et voir si ces différences de composition influenceraient les aptitudes technologiques et les caractéristiques organoleptiques notamment du beurre (traditionnel ou industriel) issu des laits provenant de ces deux races.

\section{Remerciements}

Les auteurs tiennent à remercier Mourad BOUROUIS, responsable du Laboratoire régional de Contrôle de la Qualité et de la Répression des Fraudes annexe d'Alger pour l'utilisation du CPG dans l'analyse des échantillons et pour son aide technique et analytique.

\section{Bibliographie}

Abbeddou S. et al., 2011. Modification of milk fatty acid composition by feeding forages and agro-industrial byproducts from dry areas to Awassi sheep. J.Dairy Sci., 94, 4657-4668.

AOAC (Association of Official Analytical Chemist), 1998. Official methods of analysis. $16^{\text {th }}$ ed. Arlington, VA, USA: AOAC. 
Assenat L., 1985. Le lait de brebis. Composition et propriétés. In : Luquet F.M., éd. Laits et produits laitiers. Vache-brebis-chèvre. Vol. 1. Cachan, France: Tec \& Doc Lavoisier, 281-294.

Barbosa E. et al., 2007. Effect of breed on fatty acids content in sheep milk. Archivos Zootec., 56 (Suppl.1), 681-686.

Barlowska J., Szwajkowska M., Litwinczuka Z. \& Krol J., 2011. Nutritional value and technological suitability of milk from various animal species used for dairy production. Compr. Rev. Food Sci. Food Saf., 10, 291302.

Barreto M.C., 2005. Lipid extraction and cholesterol quantification. J. Chem. Educ., 82, 103-104.

Biondi L. et al., 2008. Changes in ewe milk fatty acids following turning out to pasture. Small Ruminant Res., 75, 17-23.

Boichard D. et al., 2014. Déterminisme génétique de la composition en acides gras et protéines du lait des ruminants, et potentialités de sélection. INRA Prod. Anim., 27(4), 283-298.

Boutonnier J.L. \& D Dunant Cl., 1990. Crèmes, beurres et autres produits issus de la matière grasse. In : Luquet F.M., éd. Laits et produits laitiers. Vache-brebischèvre. Vol.2. $2^{\mathrm{e}}$ éd. Cachan, France: Tec \& Doc Lavoisier, 443-503.

Carta A. et al., 2008. Investigating the genetic component of fatty acid content in sheep milk. Small Ruminant Res., 79, 22-28.

Castro T. et al., 2009. Effect of dietary sources of vegetable fats on performance of dairy ewes and conjugated linoleic acid (CLA) in milk. Small Ruminant Res., 84, 47-53.

Chambon M., 1992. Matière grasse laitière. In: Karleskind A. \& Wolff J.-P., éds. Manuel des corps gras. Cachan, France : Tec \& Doc Lavoisier, 271-280.

Chilliard Y. \& Lamberet G., 1984. La lipolyse dans le lait : les différents types, mécanismes, facteurs de variation, signification pratique. Lait, 64, 544-578.

Cieslak A. et al., 2010. Enhancing unsaturated fatty acids in ewe's milk by feeding rapeseed or linseed oil. Czech J. Anim. Sci., 55(11), 496-504.

Collomb M., Bütikofer U., Maurer J. \& Sieber R., 2006. Composition en acides gras du lait de brebis produit à diverses altitudes. Rev. Suisse Agric., 38(6), 335-339.

De La Fuente L.F. et al., 2009. Factors influencing variation of fatty acid content in ovine milk. J. Dairy Sci., 92, 3791-3799.

Gavrilovie M., Maginot M.-J., Schwartz-Gavrilovie C. \& Wallach J., 1996. Manipulations d'analyse biochimique. Paris : Doin Éditeurs.

Gelé M. et al., 2014. Phénotypage et génotypage à grande échelle de la composition fine des laits dans les filières bovine, ovine et caprine. INRA Prod. Anim., 27(4), 255268.

Gerchev G. \& Mihaylova G., 2012. Milk yield and composition of sheep milk in Srednostarplaninska and
Tetevenska breeds. Biotechnol. Anim. Husbandry, 28(2), 241-251.

Gnädig S., Chardigny J.-M. \& Sebedio J.-L., 2001 . Lipides. In: Debry G., éd. Lait, nutrition et santé. Cachan, France : Tec \& Doc Lavoisier, 105-124.

Gomez-Cortes P. et al., 2009. Effect of supplementation of grazing dairy ewes with a cereal concentrate on animal performance and milk fatty acid profile. J. Dairy Sci., 9, 3964-3972.

Goudjil H. et al., 2003. Composition of cholesterol and its precursors in ovine milk. Lait, 83, 153-160.

Goudjil H. et al., 2004. Quantitative characterization of unsaturated and trans fatty acids in ewe's milk fat. Lait, 84, 473-482.

Hilali M., El-Mayda E. \& Rischkowsky B., 2011. Characteristics and utilization of sheep and goat milk in the Middle East. Small Ruminant Res., 10, 92-101.

Legarto J. et al., 2014. Effets des conduites d'élevage sur la production de lait, les taux butyreux et protéiques et la composition en acides gras du lait de vache, chèvre et brebis évaluée par spectrométrie dans le moyen infrarouge. INRA Prod. Anim., 27(4), 269-282.

Lock A.L., Sinclair L.A. \& Bauman D.E., 2005. Milk fat synthesis and its regulation in dairy sheep. In: Proceedings of the $11^{\text {th }}$ Annual great lakes dairy sheep symposium, November 3-5, 2005, Sheraton Burlington Hotel \& Conference Center, Burlington, VT, USA.

Mierlita D., Daraban S.T. \& Lup F., 2011a. Effects of breed on milk fatty acid profile in dairy ewes, with particular reference to cis-9, trans-11 conjugated linoleic acid. S. Afr. J. Anim. Sci., 41(3), 224-231.

Mierlita D. et al., 2011b. Comparative study regarding the fatty acids profile in sheep milk related to the breed and parity. Ann. Univ. Oradea Fascicula: Ecotoxicologie, Zootehnie Tehnologii Industrie Alimentară, 221-232.

Mihaylova G., Gerchev G., Moeckel P. \& Jahreis G., 2004. Comparative study on fatty acid content in milk of Tsigay and Karakachan sheep. Bulg. J. Vet. Med., 7(3), 181-187.

Moate P.J., Chalupa W., Boston R.C. \& Leant I.J., 2007. Milk fatty acids. I. Variation in the concentration of individual fatty acids in bovine milk. J. Dairy Sci., 90, 4730-4739.

Morand-Fehr P., Fedele V., Decandia M. \& Le Frileux Y., 2007. Influence of farming and feeding systems on composition and quality of goat and sheep milk. Small Ruminant Res., 68, 20-34.

Mordret F., 1992. Analyse des acides gras. In : Karleskind A. \& Wolff J.-P., eds. Manuel des corps gras. Cachan, France : Tec \& Doc Lavoisier, 1158-1170.

Naudet N. \& Hautfenne A., 1986. Méthode normalisée pour la détermination des stérols totaux dans les huiles et graisses. Rev. Fr. Corps Gras, 33, 167.

Paccalin J. \& Galantier M., 1986. Valeur nutritionnelle du lait et des produits laitiers. In : Luquet F.M., éd. Laits et produits laitiers. Vache-brebis-chèvre. $3^{\mathrm{e}}$ éd. Cachan, France : Tec \& Doc Lavoisier, 93-122. 
Park Y.W., Juarez M., Ramos M. \& Haenlein G.F.W., 2007. Physico-chemical characteristics of goat and sheep milk. Small Ruminant Res., 68, 88-113.

Perea S. et al., 2000. Seasonal changes in the fat composition of Lacha sheep's milk used for Idiazabal cheese manufacture. Eur. Food Res. Technol., 210, 318-323.

Pirisi A., Lauret A. \& Dubeuf J.P., 2007. Basic and incentive payments for goat and sheep milk in relation to quality. Small Ruminant Res., 68, 167-178.

Sanz Sampelayo M.R., Chilliard Y., Schmidely Ph. \& Boza J., 2007. Influence of type of diet on the fat constituents of goat and sheep milk. Small Ruminant Res., 68, 42-63.

Schmidely P. \& Sauvant D., 2001. Taux butyreux et composition de la matière grasse du lait chez les petits ruminants: effet de l'apport de matières grasses ou d'aliment concentré. INRA Prod. Anim., 14(5), 337-354.

Scintu M.F. \& Piredda G., 2007. Typicity and biodiversity of goat and sheep milk products. Small Ruminant Res., 68, 221-231.

Signorelli F. et al., 2008. Breed differences in sheep milk fatty acid profiles: opportunities for sustainable use of animal genetic resources. Small Ruminant Res., 78, 2431.

Talpur F.N., Bhanger M.I., Khooharo A.A. \& Memon G.Z., 2008. Seasonal variation in fatty acid composition of milk from ruminants reared under the traditional feeding system of Singh, Pakistan. Livest. Sci., 118, 166-173.

Talpur F.N., Bhanger M.I. \& Memon N.N., 2009. Milk fatty acid composition of indigenous goat and ewe breeds from Sindh, Pakistan. J. Food Compos. Anal., 22, 59-64.

Ulbricht T. \& Southgate D., 1991. Coronary heart disease: seven dietary factors. Lancet, 338, 985-992.

Valvo M.A., Bella M., Scerra M. \& Biondi L., 2007. Effects of ewe feeding system (grass $v s$ concentrate) on milk fatty acid composition. CIHEAM Options Méditerranéennes Ser. A, 74, 227-231.

Yabrir B. et al., 2013. Composition and nitrogen distribution of Ouled-Djellal and Rumbi Algerian ewe's milk. Adv. J. Food Sci. Technol., 5(9), 1220-1226.

Yabrir B. et al., 2014. Nutritional value of Algerian breed ewe's milk related to its mineral content. Pak. J. Nutr., 13(3), 176-180.

Zhang R.H., Mustafa A.F., Ng-Kwai-Hang K.F. \& Zhao X., 2006. Effects of freezing on composition and fatty acid profiles of sheep milk and cheese. Small Ruminant Res., 64, 203-210.

(48 réf.) 